\title{
Mapping and Exome Sequencing Identifies a Mutation in the IARS Gene as the Cause of Hereditary Perinatal Weak Calf Syndrome
}

\author{
Takashi Hirano ${ }^{1,2 *}$, Naohiko Kobayashi ${ }^{3}$, Tamako Matsuhashi ${ }^{3}$, Daisaku Watanabe ${ }^{4}$, Toshio Watanabe ${ }^{5}$, \\ Akiko Takasuga $^{5}$, Mayumi Sugimoto ${ }^{5}$, Yoshikazu Sugimoto ${ }^{1}$
}

1 Shirakawa Institute of Animal Genetics, Fukushima, Japan, 2 Laboratory of Animal Physiology, Faculty of Agriculture, Tokyo University of Agriculture, Kanagawa, Japan, 3 Hida Beef Cattle Research Department, Gifu Prefectural Livestock Research Institute, Gifu, Japan, 4 School of Veterinary Medicine and Animal Science, Kitasato University, Aomori, Japan, 5 National Livestock Breeding Center, Fukushima, Japan

\begin{abstract}
We identified an IARS (isoleucyl-tRNA synthetase) c.235G >C (p.Val79Leu) substitution as the causative mutation for neonatal weakness with intrauterine growth retardation (perinatal weak calf syndrome). In Japanese Black cattle, the syndrome was frequently found in calves sired by Bull A. Hence, we employed homozygosity mapping and linkage analysis. In order to identify the perinatal weak calf syndrome locus in a 4.04-Mb region of BTA 8, we analysed a paternal half-sibling family with a BovineSNP50 BeadChip and microsatellites. In this critical region, we performed exome sequencing to identify a causative mutation. Three variants were detected as possible candidates for causative mutations that were predicted to disrupt the protein function, including a G>C (p.Val79Leu) mutation in IARS c.235. The IARS c.235G >C mutation was not a homozygous risk allele in the 36 healthy offspring of Bull A. Moreover, the IARS Val79 residue and its flanking regions were evolutionarily and highly conserved. The IARS mutant (Leu79) had decreased aminoacylation activity. Additionally, the homozygous mutation was not found in any of 1526 healthy cattle. Therefore, we concluded that the IARS c.235G $>C$ mutation was the cause of hereditary perinatal weak calf syndrome.
\end{abstract}

Citation: Hirano T, Kobayashi N, Matsuhashi T, Watanabe D, Watanabe T, et al. (2013) Mapping and Exome Sequencing Identifies a Mutation in the IARS Gene as the Cause of Hereditary Perinatal Weak Calf Syndrome. PLoS ONE 8(5): e64036. doi:10.1371/journal.pone.0064036

Editor: Claire Wade, University of Sydney, United States of America

Received November 2, 2012; Accepted April 8, 2013; Published May 21, 2013

Copyright: (c) 2013 Hirano et al. This is an open-access article distributed under the terms of the Creative Commons Attribution License, which permits unrestricted use, distribution, and reproduction in any medium, provided the original author and source are credited.

Funding: This work was supported by grants from the Japan Racing and Livestock Promotion Foundation. The funders had no role in study design, data collection and analysis, decision to publish, or preparation of the manuscript.

Competing Interests: The authors have declared that no competing interests exist.

*E-mail: t3hirano@nodai.ac.jp

\section{Introduction}

The incidence of perinatal mortality in Japanese Black cattle is $4.5 \%$, with $27.7 \%$ of the cases caused by neonatal weakness, and without any apparent clinical symptoms. In total, $72 \%$ of neonatal deaths caused by neonatal weakness are associated with normal gestation periods and low birth weights. This suggests intrauterine growth retardation; also known as perinatal weak calf syndrome [1]. Moreover, calves with this syndrome exhibit anaemia, depression, weakness, variable body temperature, astasia, difficulty nursing, growth retardation, and increased susceptibility to infection [2]. The pathological features of perinatal weak calf syndrome are anaemia with bone marrow dysfunction and foetoplacental dysfunction. The incidence of perinatal weak calf syndrome is dependent on the paternal and maternal family, and genetic factors have been implicated [1]. However, no genetic factors have been identified as the cause of perinatal weak calf syndrome.

Of the 538,111 Japanese Black calves born in 2010, the number of calves that lived less than 3 months was 22,020 (4.1\%); in Holstein calves, the rate was $6.3 \%(34,182 / 538,656)$, according to a Japanese bovine individual identification database administered by the National Livestock Breeding Center (https://www.id.nlbc. go.jp/html/oshirase_back.html\#2012012001). In offspring sired by Bull A, we found that the frequency of calf mortality before the age of 3 months was high $(8.4 \% ; 45 / 526)$ and diagnosed them with perinatal weak calf syndrome. Bull A was not a carrier of any known genetic diseases in Japanese Black cattle (e.g., BAND3 deficiency, factor XIII deficiency, claudin-16 deficiency, molybdopterin cofactor sulfurase deficiency, Chediak-Higashi syndrome, factor XI deficiency, and Marfan syndrome-like disease) [3-9]. Thus, unknown genetic factors were implicated in the high frequency of death in calves sired by Bull A.

Next-generation sequencing technology has enabled researchers to identify variants in individuals by whole-genome resequencing [10]. Recently, DNA sequence capture techniques have made it possible to determine sequences of exons and their flanking regions by whole-exome sequencing. The Human Gene Mutation Database (http://www.homd.org) shows that $95.1 \%$ of mutations are missense/nonsense mutations, splice site mutations, and small indels in coding regions of nuclear genes that underlie or are associated with inherited disease. In humans, protein-coding sequences constitute less than $2 \%$ of the whole genome. Wholeexome sequencing has been used to analyze human genetic diseases because it is efficient and cost-effective [11-14]. Therefore, we used whole-exome sequencing, which identifies variants from exons and their flanking regions, to identify the causative mutation for perinatal weak calf syndrome. To perform 
whole-exome sequencing, we prepared a bovine whole-exon capture custom array (Nimblegen) that targeted 174,377 exons. This approach enabled us to identify the causative mutation responsible for the hereditary perinatal weak calf syndrome.

To efficiently remove genetic defects from livestock populations such as cattle, it is important to establish a mating control based on the results of DNA tests that detect disease-caused mutations. We used mapping and exome sequencing to identify the mutation responsible for perinatal weak calf syndrome.

\section{Results and Discussion}

\section{A Critical Region Detected in BTA 8}

To identify the critical region for perinatal weak calf syndrome, we constructed a paternal half-sibling family of offspring sired by Bull A. Fourteen calves with birth weights less than $20 \mathrm{~kg}$ despite normal gestation periods, weakness, and difficulty nursing were selected as the affected animals from among the calves that died before the age of 3 months (Fig. 1). A great-grandsire of Bull A was found in the fourth generation of the maternal ancestors of these calves, which suggested that perinatal weak calf syndrome was a recessive disorder.

To perform homozygosity mapping, we utilized the paternal half-sibling family. This comprised of 13 affected calves and 30 normal cattle sired by Bull A. The paternal half-sibling family was genotyped with the BovineSNP50 BeadChip. Homozygosity mapping was performed with 13,208 SNPs located in autosomes that were heterozygous in Bull A. The specific homozygous region in the affected offspring was detected on the distal half of BTA 8, at around $90 \mathrm{Mb}$ (Figs. 2A and 2B). Furthermore, we performed a linkage analysis with 14 affected calves, 36 normal cattle, and 24 informative microsatellite markers covering BTA 8. The same region was repeatedly detected as the causative region $(1 \%$ chromosome-wise significance level). The 95\% confidence interval was calculated as the region from $82.9 \mathrm{cM}$ to $103.6 \mathrm{cM}$ flanked by IDVGA-52 and $M N B-38$ (20.7-cM interval) (Fig. 2G). The IDVGA52 and $M \mathcal{N} B-38$ positions in btau4.0 were $79.3 \mathrm{Mb}$ and $102.6 \mathrm{Mb}$, indicating that the interval corresponded to the homozygosity mapping results. Based on the BovineSNP50 BeadChip genotyping, 11 of 13 affected calves $(84.6 \%)$ shared the homozygous haplotype from $87,043,929$ bp to $90,609,372$ bp flanked by $A R S$ $B F G L-N G S-108358$ and ARS-BFGL-NGS-28685 on BTA 8.

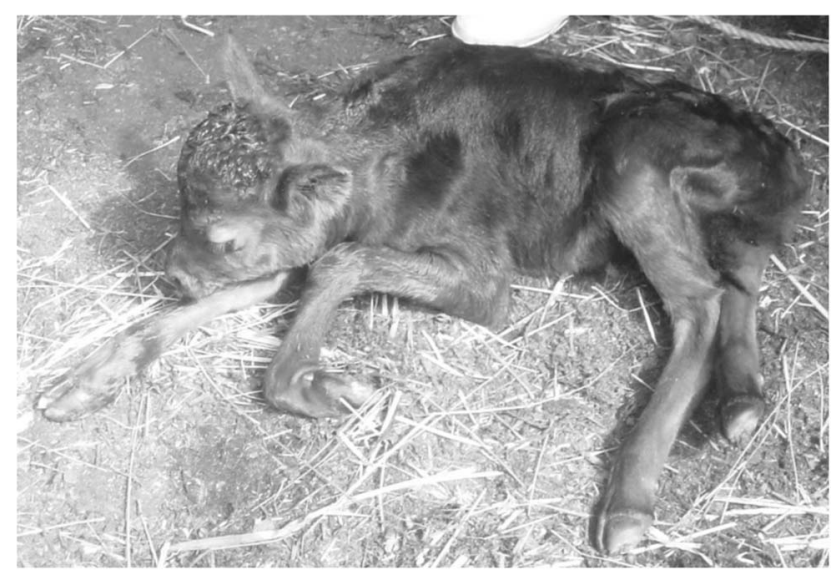

Figure 1. A calf with perinatal weak calf syndrome. The birth weight was $16 \mathrm{~kg}$ (normal average; $28.4 \pm 4.24 \mathrm{~kg}$ ). The gestation period was 296 days (normal average; 288.9 days). The affected calf was weak and had difficulty nursing. The calf died at 2 days old. doi:10.1371/journal.pone.0064036.g001
Therefore, we determined that the region flanked by these SNPs (4.04 Mb) was the critical region (Fig. 3). These findings indicated that the syndrome found in calves sired by Bull A was an autosomal recessive disorder. The mapping results provided the first evidence of a defective phenotype in perinatal weak calf syndrome that was clearly classified as an inherited disorder. This suggests that the phenotype of the remaining 2 calves among the 13 affected calves was caused by different pathogenic factors.

\section{Exome Sequencing Detects Causative Mutation Candidates}

The critical region spanned $4.04 \mathrm{Mb}$ and encompassed 29 annotated genes. To identify a causative mutation from these genes, we performed exome sequencing with 2 risk-haplotype homozygous affected calves (Affected-2, 10), 1 risk-haplotype heterozygous normal animal (Normal-1), and 1 no risk-haplotype normal animal (Normal-2) (Fig. 3). Each sample was applied to 2 lanes of a flow cell, and 1 paired-end 40-bases read length run was performed. The yield bases for each sample were Affected-2, 4,666.7 Mb (110.6×); Affected-10, 4,895.1 Mb (116.0×); Normal1, 4,894.4 Mb (116.0×); and Normal-2, 5,049.3 Mb (119.7×). Potential sequence changes including single-nucleotide variations (SNVs) and small insertions or deletions (indels) were discovered with these data reads (Affected-2; 194 SNVs and 18 indels, Affected-10; 195 SNVs and 14 indels, Normal-1; 262 SNVs and 13 indels, and Normal-2; 222 SNVs and 12 indels). To distinguish potentially pathogenic mutations from these SNVs and indels, we first selected some variants that were detected as homozygous in 1 or 2 of the affected calves and undetected in both normal animals. Then non-synonymous mutations, predicted as damaging with Polyphen-2 or SIFT, were selected. Indels causing frame shifts were not detected. This occurred because it was suggested that the point mutations at position +5 of the $5^{\prime}$ splice site were particularly prone to aberrant splicing [15]. Intronic variants located within 6 bases of the exon-intron junction were also selected. Under these criteria, $3 \mathrm{SNV}$ s were detected. Sanger sequencing confirmed that these SNVs produced the expected genotype for Bull A and 4 animals used for exome sequencing (Table 1). These SNVs, IARS c.235G $>$ C (p.Val79Leu) (NM_001101069.1), LOC786526 c.3449A $>$ G (p.Gln1150Arg) (XM_001254184.3), and CENPP c.663+3A $>$ G (NM_001105615.1), were used for further analysis.

\section{Identification of the Causative Mutation}

Considering the perinatal weak calf syndrome found in calves sired by Bull A was an autosomal recessive disorder, the causative mutation had to be homozygous for a risk allele in the affected calves. To determine a possible causative mutation from the 3 SNVs, 14 affected and 36 normal cattle used in the mapping were genotyped for 3 candidate causative SNVs. Only the IARS c. $235 \mathrm{G}>\mathrm{C}$ (p.Val79Leu) substitution was not detected as homozygous risk-allele " $C$ ' in normal animals (Table 2) and remained as a possible causative mutation. To determine whether the IARS c. $235 \mathrm{G}>\mathrm{C}$ substitution was closely linked to a causative mutation that could not be detected by exome sequencing, we sequenced all 33 exons of IARS with Sanger sequencing. Two novel heterozygous, non-synonymous SNVs were detected in Bull A: c.2435A $>$ G (p.Glu812Gly) and c.3394C $>$ T (p.Leu1132Phe). These amino acid substitutions were predicted as not damaging by PolyPhen-2 and SIFT. Furthermore, risk-allele homozygous animals for the two SNVs were identified from the 36 normal cattle (Table 2). Therefore, we determined that c.2435A $>\mathrm{G}$ and c.3394C $>$ T SNVs were not the causative mutation.

The bovine IARS gene (isoleucyl-tRNA synthetase) encodes a protein containing 1,262 amino acids. Val79 is highly conserved, 

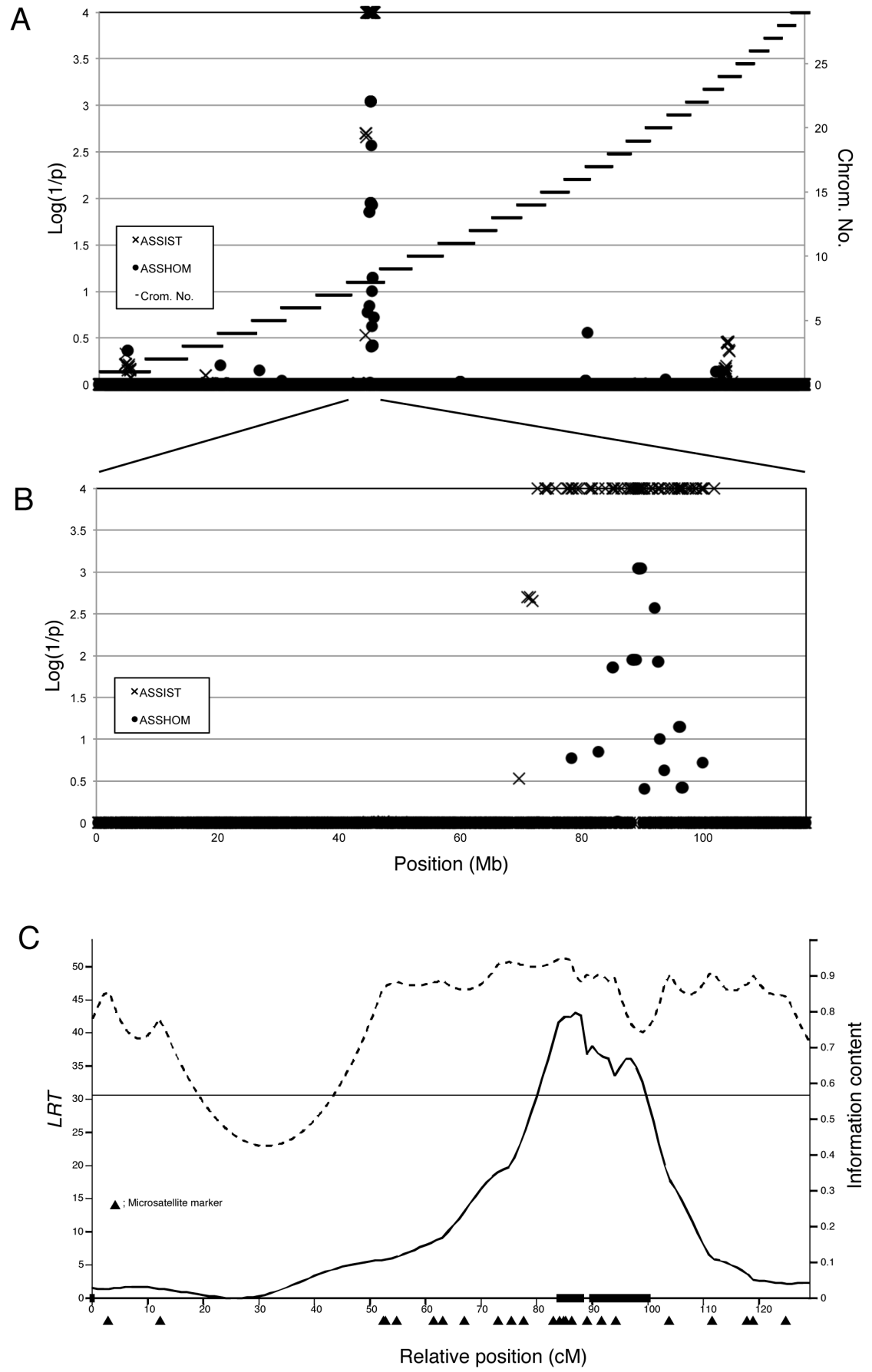

Figure 2. Genome-wide homozygosity mapping of perinatal weak calf syndrome. (A) The results of the genome-wide homozygosity mapping of perinatal weak calf syndrome with a paternal half-sib family composed of 13 affected and 30 normal animals sired from Bull A using ASSHOM (O) and ASSIST (X). The black horizontal bars mark the limits between the 29 autosomes. (B) The results of the homozygosity mapping on BTA 8. (C) The $L R T$-statistic profile for the disease on BTA 8. The horizontal line indicates the threshold for the $1 \%$ chromosome-wise significance level. The dashed line indicates information content (right $y$-axis). Microsatellite positions are indicated as filled triangles under the $x$-axis, respectively. The filled boxes on the $x$-axis represent the $95 \%$ confidence interval. The $95 \%$ confidence interval was flanked by IDVGA-52 (79.3 Mb) and MNB-38 $(102.6 \mathrm{Mb})$, corresponding to the results of homozygosity mapping.

doi:10.1371/journal.pone.0064036.g002

and the region flanking Val79 is also highly conserved in mammals (Fig. 4A). Val79 is located in a catalytic core domain of IARS (The Conserved Domain Database, NCBI). To determine the effect of p.Val79Leu mutation on IARS aminoacylation activity, we purified recombinant wild-type and mutant IARS proteins tagged with V5 and His (Fig. 4B). The aminoacylation activity of the mutant IARS protein was decreased to $38.0 \%\left(p=2.15 \times 10^{-12}\right)$ (Fig. 4C). A mixture of equal amounts of wild-type and mutant IARS proteins demonstrated the expected combined activity $(p=0.42)$, suggesting that the mutant protein was not dominantnegative. Reveromycin A $(1 \mu \mathrm{g} / \mathrm{ml})$, an IARS-specific inhibitor [16], completely inhibited the aminoacylation activity of the wild- 
Table 1. Confirmation of 3 SNPs in CENPP, IARS and LOC786526.

\begin{tabular}{|c|c|c|c|c|c|}
\hline & Bull A & $\begin{array}{l}\text { Affected- } \\
2\end{array}$ & $\begin{array}{l}\text { - Affected- } \\
10\end{array}$ & Normal-1 & 1 Normal-2 \\
\hline MS haplotype & $R /$ nonR & $R / R$ & $R / R$ & $\mathrm{R} / \mathrm{nonR}$ & $\begin{array}{l}\text { nonR/ } \\
\text { nonR }\end{array}$ \\
\hline CENPP c.663+3A>G & $A / G$ & $\mathrm{G} / \mathrm{G}$ & $\mathrm{G} / \mathrm{G}$ & $A / G$ & $A / A$ \\
\hline IARS c. $235 \mathrm{G}>\mathrm{C}$ & $\mathrm{G} / \mathrm{C}$ & $\mathrm{C} / \mathrm{C}$ & $\mathrm{C} / \mathrm{C}$ & $\mathrm{G} / \mathrm{C}$ & $\mathrm{G} / \mathrm{G}$ \\
\hline$L O C 786526$ c.3449A $>G$ & $\mathrm{~A} / \mathrm{G}$ & G/G & $\mathrm{G} / \mathrm{G}$ & $A / G$ & $A / A$ \\
\hline
\end{tabular}

$\mathrm{R}$; risk haplotype.

nonR; non-risk haplotype.

doi:10.1371/journal.pone.0064036.t001

type and mutant IARS. The decreased aminoacylation activity caused by the p.Val79Leu mutation may have contributed to the incidence of perinatal weak calf syndrome.

Moreover, there were no risk-allele homozygous $C C$ animals among the 1,526 normal cattle (146 offspring sired by Bull A and 1,380 healthy cattle unrelated to Bull A) (Table 3). In the normal population unrelated to Bull A, 194 of 1,380 (14.06\%) animals were heterozygous. In this population, the frequency of the risk allele $C$ was $7 \%$, and it was expected that 7 animals $(0.49 \%)$ among the normal cattle population would be the risk allele homozygous. However, homozygous $C C$ animals were not detected in normal cattle. Moreover, a $\chi^{2}$ test revealed that the genotypic frequencies deviated from the Hardy-Weinberg equilibrium $(p<0.01)$. These findings indicated that the risk allele homozygous $C C$ genotype was specifically detected in the affected calves and suggested that these homozygous calves died or were culled because they did not exhibit normal growth. Thus, we concluded that IARS c. $235 \mathrm{G}>\mathrm{C}$ (p.Val79Leu) is the causative mutation for hereditary perinatal weak calf syndrome in the Bull A family.

The homozygous $C C$ mutants might die during pregnancy. The FANCI deletion compromised fertility in Holstein-Friesian cattle; pregnancy failure was illustrated as the dams returned to estrus 56-270 days after insemination [17]. Artificial insemination (AI) is performed on dams in estrus, and the $\mathrm{AI}$ interval can appear as the
Table 2. Genotyping with the Bull A family used for mapping.

\begin{tabular}{|c|c|c|c|c|c|c|c|}
\hline & \multicolumn{3}{|c|}{ Affected } & \multicolumn{3}{|c|}{ Normal } & \multirow[b]{2}{*}{ Total } \\
\hline & $\mathbf{R} / \mathbf{R}$ & R/nonR & $\begin{array}{l}\text { nonR/ } \\
\text { nonR }\end{array}$ & $\mathbf{R} / \mathbf{R}$ & $R /$ nonR & $\begin{array}{l}\text { nonR/ } \\
\text { nonR }\end{array}$ & \\
\hline $\begin{array}{l}\text { CENPP } \\
\text { c. } 663+3 A>G\end{array}$ & 12 & 1 & 1 & 3 & 27 & 4 & 48 \\
\hline IARS c.235G $>C$ & 11 & 1 & 1 & 0 & 21 & 15 & 49 \\
\hline $\begin{array}{l}L O C 786526 \\
\text { c. } 3449 A>G\end{array}$ & 12 & 1 & 1 & 6 & 22 & 7 & 49 \\
\hline IARS c. $2435 \mathrm{~A}>\mathrm{G}$ & 12 & 1 & 1 & 6 & 25 & 3 & 49 \\
\hline IARS c.3394C >T & 12 & 1 & 1 & 8 & 23 & 3 & 49 \\
\hline
\end{tabular}

interval from insemination to estrus. To determine whether the IARS mutation contributed to the death of an embryo or a fetus during pregnancy, we classified 2,597 AI intervals (60-365 days) according to the genotypes of the bull and dam. The frequency of AI intervals of 60-210 days was 66 of $311(21.22 \%)$ in crosses between carrier bull $\times$ carrier dam, and 369 of 2,286 (16.14\%) for others crosses, between carrier bull $\times$ normal dam, normal bull $\times$ carrier dam and normal bull $\times$ normal dam. The frequency of a 60- to 210-day AI interval in carrier bull $\times$ carrier dam was significantly higher $(p<0.05)$ than the frequency in other crosses. Therefore, the IARS c. $235 \mathrm{G}>\mathrm{C}$ mutation may also have contributed to the death of an embryo or a fetus during pregnancy.

\section{DNA Test for Detecting the c.235G >C Mutation by Using the PCR-RFLP Method}

The IARS mutant allele $\mathrm{C}$ frequency was $7 \%$ in the normal population unrelated to Bull $\mathrm{A}$. A variant with $>5 \%$ minor allele frequency is classified as a common variant [18]; thus it appears that the mutant allele commonly exists in Japanese Black cattle. To efficiently remove the mutation from Japanese Black cattle, a DNA-based test is needed. The c. $235 \mathrm{G}>\mathrm{C}$ mutation disrupted the HincII site, preventing the mutant allele sequence from being

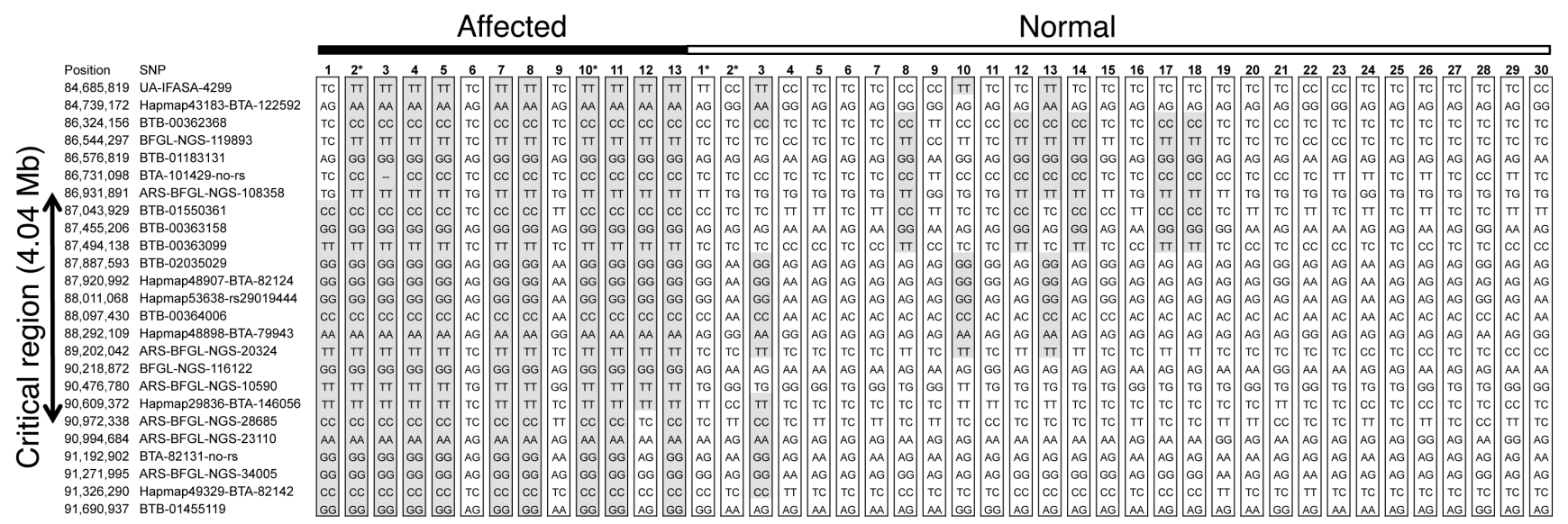

Figure 3. Genotypes of affected and normal animals for SNPs on BTA8. Overlapping blocks of extended homozygosity in the affected animals are shown in grey. The interval flanked by ARS-BFGL-NGS-108358 and ARS-BFGL-NGS-28685 (4.04 Mb) with shared homozygosity in 11/13 affected animals is determined as the critical region. In normal animals, grey blocks indicate the same homozygous haplotype shown in the affected animals with at least 6 SNPs.

doi:10.1371/journal.pone.0064036.g003 
Cattle MUT
$\nabla$

Cattle WT

YGHILAGTIKDIVTRYAHOSGF YLRRFGWDCHGLPVEYEIDKTLGIRGPEDVAKLGIAE YGHILAGTIKDIVTRYAHOSGF FVRRFGWDCHGLPVEYEIDKTLGIRGPEDVAKLGIAE

Human

YGHILAGTIKDIVTRYAHOSGF FVPRRFGWDCHGLPVEYEIDKTLGIRGPEDVAKMGITE

Chimpanzee

Gibbon

YGHILAGTIKDIVTRYAHQSGF FV PRRFGWDCHGLPVEYE IDKTLGIRGPEDVAKMGITE

Mouse

YGHILAGTIKDIVTRYAHOSGF FVRRFGWDCHGLPVEYEIDKTLGIRGPEDVARMGITE

Rat

YGHILAGTIKDIVTRYAHQSGF FVRRFGWDCHGLPVEYE IDKTLGIKGPEDVAKMGIAE

Dog YGH ILAGTIKDIVTRYAHOSGF FVRRFGWDCHGLPVEYE IDKTLGIKGPEDVAKMGIAE

Horse YGHILAGTIKDIVTRYAHQSGF FVRRFGWDCHGLPVEYEIDKTLGIRGPEDVAKMGIVE Giant Panda YGHILAGTIKDIVTRYAHQSGF FVRRFGWDCHGLPVEYEIDKTLGIRGPEDVAKMGIVE Rabbit

Chicken

Xenopus YGHILAGTIKDIVTRYAHOSGF FVPRRFGWDCHGLPVEYE IDKTLGIKGPEDVAKMGIAE

Zebrafish

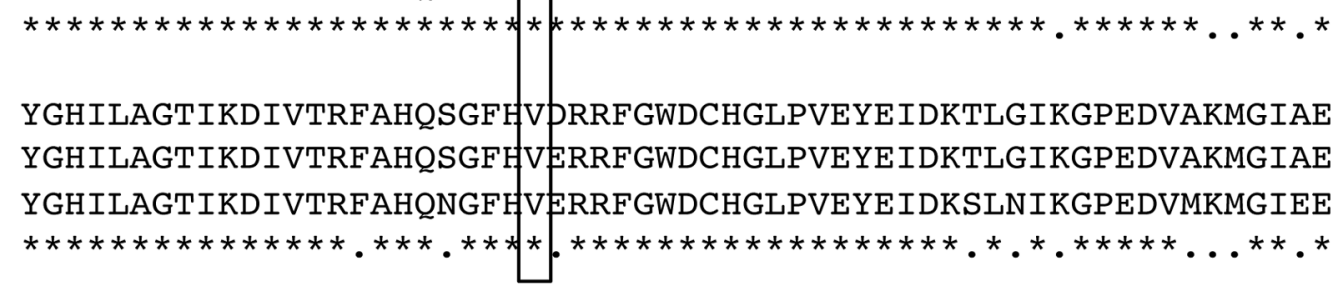

B

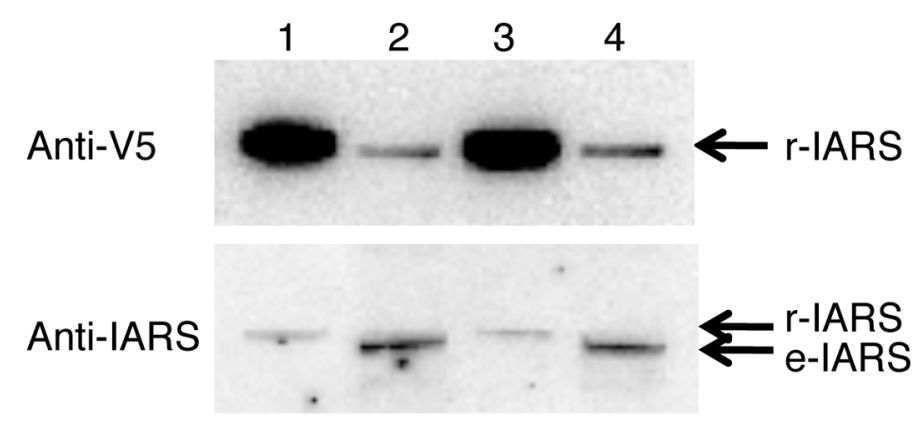

C

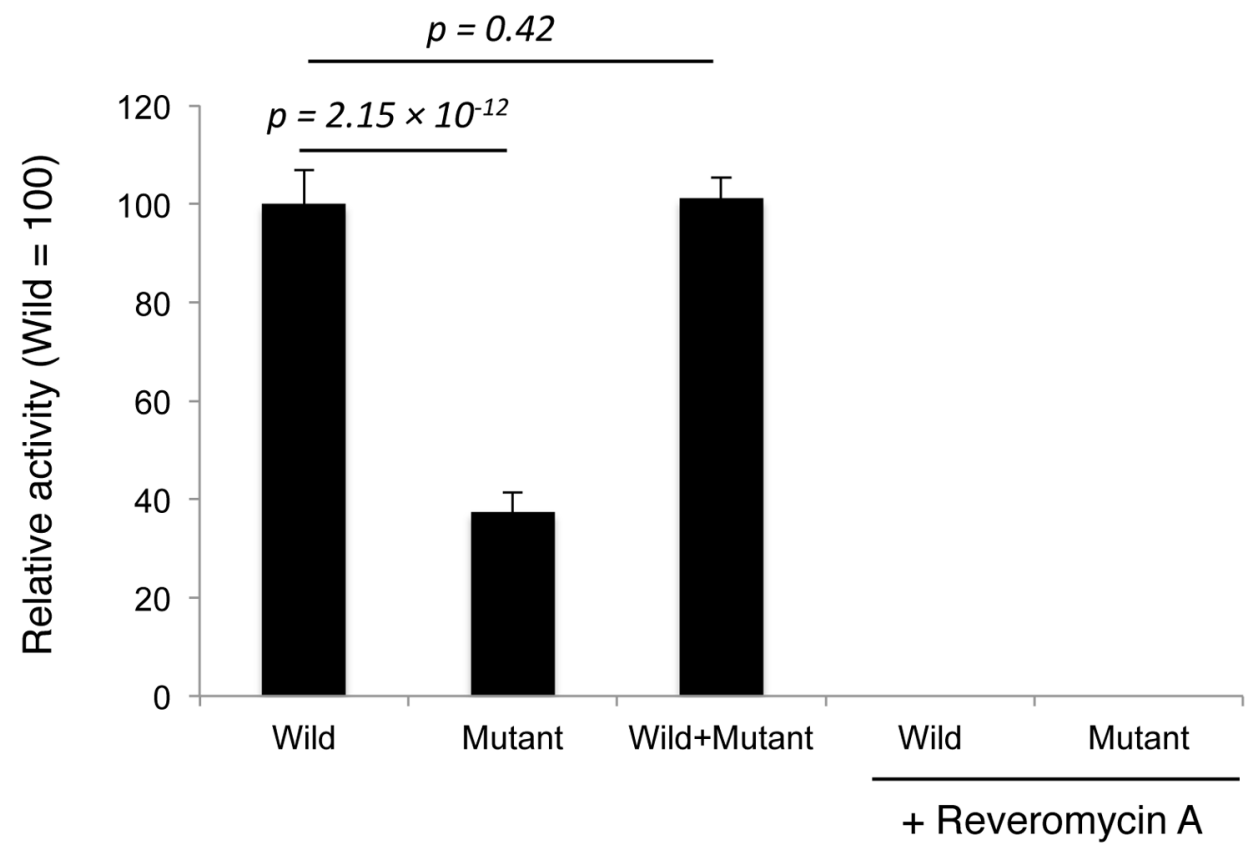


Figure 4. Alignment of the region flanking Val79Leu, and IARS aminoacylation activity. (A) Val79 is extremely conserved, and the flanking regions are also conserved in mammals. Top: cattle mutant and wild-type sequences. Middle: other mammalian sequences. Below: non-mammalian sequences. (B) NIH 3 T3 cells were transfected with wild-type (Val-79) and mutant (Leu-79) V5-His-IARS cDNA plasmids. Whole-cell lysates were treated with His-tagged protein-binding magnetic beads (Dynabeads TALON). Immunoblot analysis was performed with anti-V5 antibody (Invitrogen) and anti-IARS antibody (H-219, Santa Cruz). 1. Purified wild-type V5-His-IARS. 2. Wild-type V5-His-IARS-transfected whole-cell lysate treated with TALON beads. 3. Purified mutant V5-His-IARS. 4. Mutant V5-His-IARS-transfected whole-cell lysate treated with TALON beads. Most of the V5-His-tagged protein was absorbed by TALON beads. The molecular weights of recombinant V5-His-IARS proteins (r-IARS) were higher than the endogenous murine IARS protein (e-IARS). The $r$-IARS fractions did not contain detectable e-IARS. (C) The aminoacylation activity of purified $r$-IARS ( $n=3$, in triplicate) shown with the standard deviation; $100 \%$ corresponds to $1.22 \mathrm{pmol} / \mu \mathrm{g}$-protein, $30 \mathrm{~min}$. Revelomycin A, $1 \mu \mathrm{g} / \mathrm{ml}$. A t-test was conducted to obtain the $p$ values.

doi:10.1371/journal.pone.0064036.g004

digested by HincII. Therefore, we developed a DNA-based test for detecting the mutation by using polymerase chain reactionrestriction fragment length polymorphism (PCR-RFLP) (Fig. S1).

Pinna tissues were collected from 200 calves that died before the age of 3 months, without any criteria for birth weight, weakness or difficulty nursing. These samples were genotyped for the c. $235 \mathrm{G}>\mathrm{C}$ mutation, and 41 of $200(20.5 \%)$ animals were homozygous $C C$ (Table 4). Therefore, it is expected that the DNA test may decrease the number of calf mortality before the age of 3 months by $20.5 \%$. Moreover, 19 homozygous $C C$ animals $(34.5 \%)$ were detected in a population of 55 cattle with growthretardation sired by heterozygous $G C$ Bull B (Table 4). The sire of Bull B was the great-grandsire of Bull A. It is also possible that the DNA test may be useful for preventing the incidence of growth retardation.

Aminoacyl-tRNA synthetases (ARSs) are ubiquitously expressed enzymes that are essential for the first step of protein synthesis. The aetiology of a number of diseases is connected to specific ARSs [19]. Mutations in GARS, YARS, AARS and KARS cause Charcot-Marie-Tooth (CMT) disease [20-23], and mutations in DARS2 are responsible for leukoencephalopathy with brain stem and spinal cord involvement and lactate elevation (LBSL) [24]. The SARS2 mutation was identified as a pathogenic cause of hyperuricaemia, pulmonary hypertension, renal failure in infancy, and alkalosis (HUPRA syndrome) [25]. A reduction of the aminoacylation activity of these mutant ARS proteins was shown and predicted. However, some GARS mutant proteins had full aminoacylation activity, this suggested that aminoacylation was not a fundamental cause of CMT disease [26]. The functional versatility of ARSs is being considered as a possible pathogenesis mechanism, but the role of tRNA synthetases in disease pathogenesis remains unclear [19]. Although the decrease in aminoacylation activity was detected in IARS mutants, a disruption of other possible IARS functions may contribute to the occurrence of hereditary perinatal weak calf syndrome.

Table 3. Further genotyping of IARS mutations.

\begin{tabular}{lllll}
\hline & \multicolumn{2}{l}{ Sired by Bull A } & & Unrelated \\
\cline { 2 - 3 } c.235G $>$ C & Affected & Normal & Normal \\
\hline G/G & 1 & 71 & 1,186 \\
G/C & 1 & 75 & 194 \\
C/C & 13 & 0 & 0 \\
Total & 15 & 146 & 1,380 \\
C Freq. & 0.9 & 0.26 & 0.07 \\
\hline doi:10.1371/journal.pone.0064036.t003 & &
\end{tabular}

\section{Materials and Methods}

\section{Ethics Statement}

Blood, semen or tissue samples were collected from bulls, dams, cattle, and calves by trained veterinarians following standard procedures and relevant national guidelines. The animal owners agreed that the samples could be used for our study. All animal experimentation was undertaken with the approval of the Commission of Shirakawa Institute of Animal Genetics (H20 - 8).

\section{Animal Samples}

Bull A, 14 perinatal weak calf syndrome-affected calves and 146 normal cattle sired by Bull A, Bull B, 55 growth-retarded cattle sired by Bull B, 200 calves that died before the age of 3 months and 1,380 normal cattle unrelated to Bull A were used for the analysis. Calves with a birth weight less than $20 \mathrm{~kg}$ (normal average birth weight; $28.4 \pm 4.24 \mathrm{~kg}$ ) despite a normal gestation period; weakness; difficulty nursing and a lifespan of less than 3 months were selected as affected. Healthy, normal-sized (carcass weight; $>300 \mathrm{~kg}$ ) that were collected in the slaughterhouse (Metropolitan Central Wholesale Market, Tokyo) were considered to be normal cattle. Cattle with a birth weight of 15-26 kg and a normal appetite were considered to have growth retardation cattle. Two hundred calves that died before the age of 3 months were included without criteria for birthweight, weakness or nursing. Genomic DNA was extracted from pinna tissues of affected calves, blood or adipose tissues of normal cattle, and the blood or semen of bulls according to standard protocols. The DNA concentration was adjusted to $100 \mathrm{ng} / \mu \mathrm{l}$ for the BovineSNP50 BeadChip assay and to $20 \mathrm{ng} / \mu \mathrm{l}$ for the microsatellite marker genotyping.

\section{Genotyping}

Whole-genome genotyping was performed with BovineSNP50 BeadChip (Illumina). For genotyping with BovineSNP50 BeadChip, the integrity and fragmentation status of genomic DNA was verified with agarose gel electrophoresis, and $400 \mathrm{ng}$ of DNA was used for the assay.

PCR conditions were optimized, as previously described [27,28], for genotyping 24 microsatellite markers covering BTA 8 , and Bull A was determined to be heterozygous at these markers. Genotyping was performed by using PCR with a fluorescencelabelled reverse primer, followed by electrophoresis with an ABI 3730 DNA Analyzer, as previously described [29]. These were analyzed with GeneMapper software (Applied Biosystems, Foster City, CA).

\section{Mapping}

Homozygosity mapping was performed with the ASSIST and ASSHOM programs [30].

The sires' haplotypes were reconstructed by using the interval mapping method for half-sibling families $[31,32]$. To test the hypothesis that a chromosomal location was related to the disease status, we used the following logistic regression model [33]: 
Table 4. IARS mutations in random dead calves and growthretarded calves.

\begin{tabular}{|c|c|c|}
\hline & Dead calves & Growth reterdation \\
\hline$c .235 G>C$ & before 3 months old ${ }^{\text {a) }}$ & Sired by Bull Bb) \\
\hline G/G & 122 & 26 \\
\hline $\mathrm{G} / \mathrm{C}$ & 37 & 10 \\
\hline $\mathrm{C} / \mathrm{C}$ & 41 & 19 \\
\hline Total & 200 & 55 \\
\hline C Freq. & 0.30 & 0.44 \\
\hline
\end{tabular}

$$
y_{i}=\frac{e^{a+b x_{i}}}{1+e^{a+b x_{i}}}
$$

where $y_{i}$ is the disease status $(0$ or 1$)$ and $x_{i}$ is the probability that the $i$ th offspring inherited the sire's first haplotype at a given chromosomal location. Parameters $a$ and $b$ were estimated at each location. The $\log$ likelihood of the alternative hypothesis and null hypothesis that $b=0$ are

$$
L_{1}=\sum_{i=1}^{n} y_{i} \log \left(p_{1 i}\right)+\sum_{i=1}^{n}\left(1-y_{i}\right) \log \left(1-p_{1 i}\right)
$$

and

$$
L_{0}=\sum_{i=1}^{n} y_{i} \log \left(p_{0 i}\right)+\sum_{i=1}^{n}\left(1-y_{i}\right) \log \left(1-p_{0 i}\right)
$$

respectively, where $p_{1 i}=\frac{e^{\hat{a}+\hat{b} x_{i}}}{1+e^{\hat{a}+\hat{b} x_{i}}}$ and $p_{0 i}=\frac{e^{\hat{a}}}{1+e^{\hat{a}}}$.

The test statistic $L R T=-2\left(L_{0}-L_{1}\right)$. To determine the thresholds of the LRT statistics for chromosome-wise and experiment-wise significance, 10,000 random permutations of the phenotypic data were performed [34]. The disease locus location with $95 \%$ confidence intervals was estimated by the bootstrap method [35].

\section{Exome Sequencing}

To perform bovine whole-exome sequencing, we prepared an exon sequence capture array (Nimblegen) covering 95\% (42.2 Mb) of the 174,377 bovine exons.

Two risk haplotype homozygous syndrome-affected calves, one non-risk haplotype normal cattle and one risk haplotype heterozygous normal animal were selected based on microsatellite reconstructed haplotypes. DNA was extracted from pinna tissues of these animals for constructing sequencing libraries. Libraries for paired-end sequencing were prepared by using a paired-end sample prep kit (Illumina) following the manufacturer's protocol. Five micrograms of DNA was fragmented by using a nebuliser, subjected to end repair, and ligated to adapters. The DNA fragments were separated by $2.0 \%$ agarose gel electrophoresis, and 300-350 bp fragments were eluted. One microgram of the constructed library was hybridized to the exon sequence capture array, and hybridized fragments were eluted as an exome sequencing library following the manufacturer's protocol (Nimblegen). Each library was applied to 2 lanes of a flow cell, and pairedend sequencing was performed with a read length of 40 bases on a Genome Analyzer IIx (Illumina). Btau4.0 was used as the bovine reference genome. The reads were aligned to btau 4.0 allowing two mismatches with GERALD.pl, and SNV and indel discovery was performed with run.pl in CASAVA software vl.7 (Illumina).

The effects of the detected missense mutations on protein function were analysed with PolyPhen-2 (http://genetics.bwh. harvard.edu/pph2/) and SIFT (http://sift.jcvi.org/) [36,37].

\section{Sanger Sequencing}

IARS c.235G $>\mathrm{G} \quad$ (p.Val79Leu), LOC786526 c.3449A $>\mathrm{G}$ (p.Gln1150Arg) and CENPP c.663+3A $>\mathrm{G}$, detected by exome sequencing, were validated with direct sequencing using the following primers: IARS: F 5'-TTACCTTCTATGATGGGCCTC-3' and R 5'-TTAACATCGCTGCGCTATGAC-3'; LOC786526: F 5'-GAGGCATAAACCAGGAGAGC-3' and R 5'-TGATCTACTTTCGACTGGTCG-3'; CENPP: F 5'AGATTTTCAGGGAATGCAAGAC-3', and R 5'-ACATCTCAAGATGTCAGTTAGG-3' .

To sequence all IARS exons, primers flanking the exons were designed for each intronic region (Table S1). PCR was performed by using the genomic DNA of Bull A.

Amplified products were directly sequenced using the Big Dye terminator kit and the ABI 3730 DNA Analyzer (Applied Biosystems).

\section{IARS Aminoacylation Activity}

Bovine wild-type and p.Val79Leu-mutated IARS cDNA sequences were prepared from bovine thymus cDNA using an Infusion Advantage PCR cloning kit (Clontech). The primers used in the preparation are shown in Table S2. The prepared cDNAs were subcloned into pcDNA3.1/V5-His-TOPO (Invitrogen). The plasmid was transfected into mouse NIH 3T3 cells with Lipofectamine 2000 (Invitrogen). The transfected cells were lysed in cold phosphate-buffered saline (PBS) containing 1\% Triton X100 and a proteinase inhibitor cocktail (Sigma-Aldrich). V5-HisIARS protein was purified with Dynabeads TALON (Invitrogen). The eluted fraction was concentrated with an Amicon ultracentrifugal filter device (Millipore) and diluted with PBS containing $0.1 \%$ Triton $\mathrm{X}-100$. The protein concentration of the purified IARS was measured by protein assay (BioRad).

The aminoacylation activity of purified V5-His-IARS was determined as previously described [38]. The $20 \mu \mathrm{l}$ reaction mixture contained $50 \mathrm{mM}$ HEPES (pH 7.4), $20 \mathrm{mM} \mathrm{KCl,} 5 \mathrm{mM}$ DTT, $5 \mathrm{mM} \mathrm{MgCl}_{2}, 4 \mathrm{mM}$ ATP, $1 \mathrm{mM}$ spermine, $1 \mathrm{mg} / \mathrm{ml}$ yeast total tRNA (Invitrogen), $0.05 \mu \mathrm{Ci} \quad \mathrm{L}-\left[{ }^{14} \mathrm{C}\right]$ isoleucine $(7.5 \mu \mathrm{M}: 334 \mathrm{mCi} / \mathrm{mmol})$, and purified IARS protein $(1.0$ $1.3 \mu \mathrm{g})$. The omission of ATP was used as a blank. Reactions were incubated with the enzyme for $30 \mathrm{~min}$ at $30{ }^{\circ} \mathrm{C}$. The rate was time dependent up to $30 \mathrm{~min}$ under the conditions. The reaction was terminated by adding $70 \mu \mathrm{l}$ of $180 \mathrm{mM} \mathrm{NaOAc/HOAc}$ (pH 3.0) and $1 \mathrm{mg} / \mathrm{ml}$ salmon sperm DNA (Sigma-Aldrich). An equal volume of $20 \%$ trichloroacetic acid (TCA) was added to precipitate the nucleic acids. A reaction tube was briefly agitated by mixing and then centrifuged for $15 \mathrm{~min}$ at $15,000 \mathrm{rpm}$ at $4^{\circ} \mathrm{C}$. The precipitate was washed three times with $200 \mu \mathrm{l}$ of $5 \%$ TCA containing $100 \mathrm{mM}$ isoleucine, followed by washing once with $95 \%$ ethanol. The precipitate was solubilised with $70 \mu \mathrm{l}$ of $100 \mathrm{mM} \mathrm{NaOH}$. Radioactivity was quantified in a scintillation counter (Beckman Coulter). 


\section{Artificial Insemination Interval Data}

To analyze whether the $I A R S$ c. $235 \mathrm{G}>\mathrm{C}$ mutation contributed to the death of an embryo or fetus, data from 2,597 artificial insemination (AI) intervals of dams that received $\mathrm{AI}$ again from 60 to 365 days after the initial AI were collected. These AI intervals were classified as crosses between carrier bull $\times$ carrier dam or crosses of other pairs, according to the genotype of the bull and dam. The frequencies of $\mathrm{AI}$ intervals shorter than 210 days in each group were analyzed by $\chi^{2}$ test.

\section{IARS c.235G >C Detection with PCR-RFLP}

Mutation detection by PCR-RFLP was performed by HincII digestion of the PCR products with the primers for IARS c.235 $\mathrm{G}>\mathrm{C}$ described above. Following HincII digestion, the normal PCR products were detected in the 276-bp and 116-bp digested fragments, and the mutant products only were detected in the 392bp uncut fragment.

\section{Supporting Information}

Figure S1 The DNA test using the PGR-RFLP method. Normal and mutant alleles can be distinguished by HincII digestion of PCR products. The undigested fragment indicates

\section{References}

1. Ogata Y, Nakao T, Takahashi K, Abe H, Misawa T, et al. (1999) Intrauterine growth retardation as a cause of perinatal mortality in Japanese black beef calves. J Vet Med A 46: 327-334.

2. Takasu M, Shirota K, Ohba Y, Nishi N, Murase T, et al. (2008) Thymic hypoplasia in Japanese black calves with perinatal weak calf syndrome. J Vet Med Sci 70: 1173-1177.

3. Inaba M, Yawata A, Koshino I, Sato K, Takeuchi M, et al. (1996) Defective anion transport and marked spherocytosis with membrane instability caused by hereditary total deficiency of red cell band 3 in cattle due to a nonsense mutation. J Clin Invest 97: 1804-1817.

4. Ogawa H (1996) Factor XIII deficiency in Japanese Black cattle. J Vet Med 49: 411-412 (In Japanese).

5. Hirano T, Kobayashi N, Itoh T, Takasuga A, Nakamaru T, et al. (2000) Null mutation of $P C L N-1 /$ Claudin-16 results in bovine chronic interstitial nephritis. Genome Res 10: 659-663.

6. Watanabe T, Ihara N, Itoh T, Fujita T, Sugimoto Y (2000) Deletion mutation in Drosophila ma-l homologous, putative molybdopterin cofactor sulfurase gene is associated with bovine xanthinuria type II. J Biol Chem 275: 21789-21792.

7. Yamakuchi H, Agaba M, Hirano T, Hara K, Todoroki J, et al. (2000) ChediakHigashi syndrome mutation and genetic testing in Japanese black cattle (Wagyu). Anim Genet 31: 13-19.

8. Kunieda M, Tsiji T, Abbasi AR, Khalaj MK, Ikeda M, et al. (2005) An insertion mutation of bovine F11 gene is responsible for factor XI deficiency in Japanese Black cattle. Mamm Genome 16: 383-389.

9. Hirano T, Matsuhashi T, Kobayashi N, Watanabe T, Sugimoto Y (2011) Identification of an FBN1 mutation in bovine Marfan syndrome-like disease. Anim Genet 43: 11-17.

10. Wheeler DA, Srinivasan M, Egholm M, Shen Y, Chen YL, et al. (2008) The complete genome of an individual by massively parallel DNA sequencing. Nature 452: 872-876.

11. Bamshad MJ, Ng BS, Bigham AW, Tabor HK, Emond MJ, et al. (2011) Exome sequencing as a tool for Mendelian disease gene discovery. Nat Rev Genet 12: $745-755$.

12. Ng SB, Buckingham KJ, Lee C, Bigham AW, Tabor HK, et al. (2010). Exome sequencing identifies the cause of a mendelian disorder. Nat Genet 42: 30-35.

13. Doi H, Yoshida K, Yasuda T, Fukuda M, Fukuda Y, et al. (2011) Exome sequencing reveals a homozygous STT14 mutation in adult-onset, autosomalrecessive spinocerebellar ataxia with psychomotor retardation. Am J of Hum Genet 89: 320-327.

14. Glazov EA, Zankl A, Donskoi M, Kenna TJ, Thomas GP, et al. (2011) Wholeexome re-sequencing in a family quartet identifies POP1 mutations as the cause of a novel skeletal dysplasia. PLoS Genet 7: e1002027.

15. Buratti E, Chivers M, Královičová J, Romano M, Baralle M, et al. (2007) Aberrant 5 ' splice sites in human disease genes: mutation pattern, nucleaotide structure and comparison of computational tools that predict their utilization. Nucleic Acids Res 35: 4250-4263.

16. Miyamoto Y, Machida K, Mizunuma M, Emoto Y, Sato N, et al. (2002) Identification of Sacharomyces cerevisiae isoleucyl-tRNA synthetase as a target of the G1-specific inhibitor reveromycin A. J Biol Chem 277: 28810-28814. the mutant allele, and digested fragments indicate the wild-type allele. Each genotype was determined by direct sequencing. The same genotypes were obtained by PCR-RFLP.

(TIF)

Table S1 Primers used to sequence all exons of the bovine IARS.

(PDF)

Table S2 Primers used to prepare the wild-type and mutant IARS cDNA.

(PDF)

\section{Acknowledgments}

The authors thank Kanako Yoshinari for technical assistance. We also thank Professor Michel Georges for providing ASSIST and ASSHOM software.

\section{Author Contributions}

Conceived and designed the experiments: TH YS. Performed the experiments: TH AT MS YS. Analyzed the data: TH MS. Contributed reagents/materials/analysis tools: TH NK TM DW TW. Wrote the paper: TH TW YS

17. Charlier C, Agerholm JS, Coppieters W, Karlskov-Mortensen P, Li W, et al. (2012) A deletion in the Bovine FANCI gene compromises fertility by causing fetal death and Brachyspina. PLoS One 7: e43085.

18. The 1000 Genome Project Consortium (2010) A map of human genome variation from population-scale sequencing. Nature 467: 1061-1073.

19. Park SG, Schimmel P, Kim S (2008) Aminoacyl tRNA synthetases and their connections to disease. Proc Natl Acad Sci U S A 105: 11043-11049.

20. Antonellis A, Ellsworth RE, Sambuughin N, Puls I, Abel A, et al. (2003) Glycyl tRNA synthetase mutation in Charcot-Marie-Tooth disease type 2D and distal spinal muscular atrophy type V. Am J Hum genet 72: 1293-1299.

21. Jordanova A, Irobi J, Thomas FP, Dijck PV, Meerschaert K, et al. (2006) Disrupted function and axonal distribution of mutant tyrosyl-tRNA sythetase in dominant intermediate Charcot-Marie-Tooth neuropathy. Nat Genet 38: 197202.

22. Latour $\mathrm{P}$, Thauvin-Robinet $\mathrm{C}$, Baudelet-Méry $\mathrm{C}$, Soichot $\mathrm{P}$, Cusin $\mathrm{V}$, et al. (2010) A major determinant for binding and aminoacylation of tRNA ${ }^{\text {Ala }}$ in cytoplasmic alanyl-tRNA synthetase is mutated in dominant axonal Charcotmarie-Tooth disease. Am J Hum genet 86: 77-82.

23. McLaughlin HM, Sakaguchi R, Liu C, Igarashi T, Pehlivan D, et al. (2010) Compound heterozygosity for loss-of-function lysyl-tRNA synthetase mutations in a patient with peripheral neuropathy. Am J Hum genet 87: 560-566.

24. Scheper CS, Klok Tvd, Andel RJv, Berkel CGMv, Sissler M, et al. (2007) Mitochondrial aspartyl-tRNA synthetase deficiency causes leukoencephalopathy with brain stem and spinal cord involvement and lactate elevation. Nat Genet 39: 534-539.

25. Belostotsky R, Ben-Shalom E, Rinat C, Becker-Cohen R, Feinstein S, et al. (2011) Mutation in the mitochondrial seryl-tRNA synthetase cause hyperuricemia, pulmonary hypertension, renal failure in infancy and alkalosis, HUPRA syndrome. Am J Hum genet 88: 193-200.

26. Nangle LA, Zhang W, Xie W, Yang XL, Schimmel P (2007) Charcot-MarieTooth disease-associated mutant tRNA synthetases linked to altered dimer interface and neurite distribution defect. Proc Natl Acad Sci U S A 104: 11239 11244.

27. Kappes SM, Keel JW, Stone RT, McGraw RA, Sonstegard TS, et al. (1997) A second-generation linkage map of the bovine genome. Genome Res 7: 235-249.

28. Ihara N, Takasuga A, Mizoshita K, Takeda H, Sugimoto M, et al. (2004) A comprehensive genetic map of the cattle genome based on 3802 microsatellites. Genome Res 14: 1987-1998.

29. Hirano T, Nakane S, Mizoshita K, Yamakuchi H, Inoue-Murayama M, et al. (1996) Characterization of 42 highly polymorphic bovine microsatellite markers. Anim Genet 27: 365-368.

30. Charlier C, Coppieters W, Rollin F, Desmecht D, Agerholm JS, et al. (2008) Highly effective SNP-based association mapping and management of recessive defects in livestock. Nat Genet 40: 449-454.

31. Haley CS, Knott SA, Elsen JM (1994) Mapping QTL in crosses between outbred lines using least squares. Genetics 136: 1195-1207.

32. Seaton G, Haley CS, Knott SA, Kearsey M, Visscher PM (2002) QTL Express: mapping quantitative trait loci in simple and complex pedigrees. Bioinformatics 18: 339-340. 
33. Xu S, Atchley WR (1996) Mapping quantitative trait loci for complex binary diseases using line crosses. Genetics 143: 1417-1424.

34. Churchill GA, Doerge RW (1994) Empirical threshold values for quantitative trait mapping. Genetics 138: 963-971.

35. Visscher PM, Thompson R, Haley CS (1996) Confidence intervals in QTL mapping by bootstrapping. Genetics 143: 1013-1020.
36. Adzhubei IA, Schmidt S, Peshkin L, Ramensky VE, Gerasimova A, et al. (2010) A method and server for predicting damaging missense mutations. Nat methods 7: 248-249.

37. Ng PC, Henikoff S (2001) Predicting deleterious amino acid substitutions. Genome Res 11: 863-874.

38. Beebe K, Waas W, Druzina Z, Guo M, Schimmel P (2007) A universal plate format for increased throughput of assays that monitor multiple aminoacyl transfer RNA synthetase activities. Anal Biochem 368: 111-121. 